\title{
«Redaktionelle Freiheit garantiert Vielseitigkeit und Qualität»
}

\section{Interview: Bruno Kesseli}

Dr. med. et lic. phil., Chefredaktor

\author{
Ludwig T. Heuss hat die Geschichte des Schweizerischen Ärzteverlags EMH an
} vorderster Front miterlebt und mitgeprägt. Nach 18 Jahren Tätigkeit für EMH, die letzten 11 davon als Präsident des Verwaltungsrates, hat er dieses Amt im Juni an Hans Kurt** übergeben und ist aus dem Führungsgremium zurückgetreten. Im Interview gibt er spannende Einblicke in die ebenso wechselvolle wie erfolgreiche Geschichte des Verlags der Schweizer Ärztinnen und Ärzte.

Siehe dazu auch das Editorial von Ludwig T. Heuss in dieser Ausgabe.

** Ein Interview mit dem neuen Verwaltungsratspräsidenten von EMH, Dr. med. Hans Kurt, folgt in einer der nächsten Ausgaben der SÄZ.

\section{Welche Gründe haben Sie dazu bewogen, EMH zum jetzigen Zeitpunkt zu verlassen und den Stab des Ver- waltungsratspräsidenten an Hans Kurt zu überge- ben?}

Wenn man für eine solche Entscheidung immer darauf wartet, bis etwas abgeschlossen und fertig ist, erwischt man den Zeitpunkt nie. Ein aktives Unternehmen ist ja nie "fertig», sondern immer in Bewegung und Veränderung. Ich durfte die bisherige Entwicklung von EMH mitgestalten, miterleben und manchmal auch miterleiden. Natürlich hätte ich mir noch dieses oder jenes Ziel vorstellen können, aber andererseits ist das Unternehmen heute personell und finanziell konsolidiert und auf gutem Kurs. Da ist es der richtige Zeitpunkt, Platz zu machen, damit neue und andere Veränderungen möglich werden. Ausserdem habe ich selbst auch Lust, etwas Neues anzufangen.

Sie haben die Geschichte des Verlags, der ja im Mehrheitsbesitz der FMH und damit der schweizerischen Ärzteschaft ist, von Anfang an miterlebt und

\section{Ludwig Theodor Heuss}

Geboren 1961 in Basel. Prof. Dr. med., Facharzt für Innere Medizin und Gastroenterologie, eMBA-HSG. Chefarzt der Medizinischen Klinik des Spitals Zollikerberg seit 2007. Zentralpräsident des VSAO 1991-1999. Mitglied des Zentralvorstands FMH 1994-2006. Leiter der Arbeitsgruppe Neuordnung der Facharzttitel 1994-1996. Leiter des Ressorts DDQ, Mitglied in eidgenössischen Expertenkommissionen zur Revision des Medizinalberufegesetzes. Seit 1992 Mitglied in vorbereitenden Kommissionen zur Gründung eines Ärzteverlages, Mitglied des Verwaltungsrates von EMH 1997-2015, 2004-2015 als Präsident. mitgeprägt, was eine kurze Rückschau nahelegt. Wie kam es überhaupt dazu, dass der Schweizerische Ärzteverlag 1997 aus der Taufe gehoben wurde? Die Anfänge gehen eigentlich bis auf eine Ad-hocArbeitsgruppe der FMH zurück, in der wir 1991/92 die Publizistik und Kommunikation der FMH untersuchten und Vorschläge zur Verbesserung der Ärztezeitung machten. Im 1994 neu gewählten Zentralvorstand nahmen wir diese Resultate auf und analysierten die vorhandenen Defizite und drohenden Gefahren sehr klar und nüchtern. Wir sahen die sehr beschränkten Gestaltungsmöglichkeiten in der damaligen SÄZ und beobachteten die zunehmende Ausbreitung ausländischer Medizinverlage in der Schweiz. Wir formulierten daher als ein wesentliches strategisches Ziel, dass die Ärzteschaft in Form der FMH eine aktiv gestaltende Rolle im Feld der medizinischen Publizistik in der Schweiz spielen solle. Das war bis dahin nicht der Fall.

\section{Im Gegensatz zu ...?}

(Lacht) Wir nahmen uns grosse Ziele und Vorbilder! Wir wollten vielleicht insgeheim etwas Ähnliches erreichen wie die British Medical Association, die als Eigentümerin der BMJ Publishing Group sowohl im gesundheitspolitischen als auch im Weiterbildungsund wissenschaftlichen Bereich eine führende Rolle im United Kingdom spielt.

Welche Rolle spielte der damalige, 2010 verstorbene FMH-Präsident Hans Heinrich Brunner im Zusammenhang mit der Verlagsgründung?

Eine ganz zentrale Rolle. Hans Heinrich erkannte sehr klar die strategische Bedeutung, die es hatte, ein «Feld 
zu besetzen", und er kam auch als Erster zu dem Schluss, dass dies nur durch die Gründung eines eigenen selbständigen Verlages geschehen könne. Er kam ja selbst auch aus einem verlegerischen Umfeld, sein Vater war Generaldirektor bei Ringier gewesen. Vor allem hatte Hans Heinrich die Beharrlichkeit, dieses Projekt auch gegen Widerstände umzusetzen.

\section{In welcher Funktion waren Sie beteiligt?}

Ich war schon Mitglied der Ad-hoc-Arbeitsgruppe und gehörte im Zentralvorstand von Anfang an zum engeren Zirkel, der mit diesem Projekt vertraut war, zusammen mit Reto Steiner, Pierre Aubert und Jean-François Deschenaux.

\section{Wie kam man auf Schwabe als Partner für die Gründung des neuen Verlags?}

Es haben sich damals mehrere Verlage um eine Kooperation beworben. Das wesentliche Argument für Schwabe war die Bereitschaft, die Schweizerische Medizinische Wochenschrift als führendes wissenschaftliches Organ in das Projekt einzubringen. Wenn man an das BMJ-Konzept denkt, liegt diese Entscheidung auf der Hand. Lustigerweise war dies sogar eine Art von Familienzusammenführung, denn historisch gesehen sind

\section{CORRESPONDENZ-BLATT}

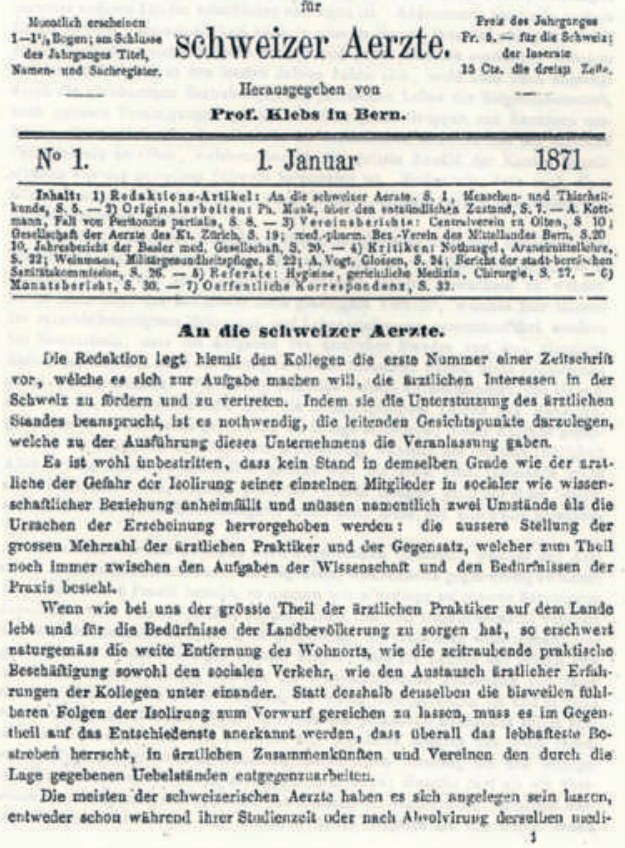

Abbildung 1: Aus dem bei Schwabe erschienenen Correspondenz-Blatt für schweizer Aerzte gingen die Schweizerische Ärztezeitung und die Schweizerische Medizinische Wochenschrift, heute Swiss Medical Weekly, hervor. beide Zeitschriften einmal aus dem ursprünglichen Correspondenz-Blatt für schweizer Aerzte entstanden, das sich erst in den 20er Jahren aufgespaltet hat (Abb.1).

«Wir formulierten daher als ein wesentliches strategisches Ziel, dass die Ärzteschaft in Form der FMH eine aktiv gestaltende Rolle im Feld der medizinischen Publizistik in der Schweiz spielen solle.»

\section{Gibt es Anekdoten aus der Gründungszeit, die Ihnen speziell in Erinnerung geblieben sind?}

Da gäbe es einige ... Zu den schöneren Erinnerungen gehört ein schöner Frühlingstag am Ufer des Vierwaldstättersees, an dem wir mit HHB [Hans Heinrich Brunner, Anm. d. Red.] Eckpunkte und Konzepte der Verlagsidee buchstäblich auf der Papierserviette skizzierten (Abb. 2). Der konkrete Gründungstermin der notariellen Vertragsunterzeichnung fiel dann zufälligerweise auf meinen Geburtstag. Da liess sich doppelt feiern.

\section{In verschiedener Hinsicht wurde mit der Gründung eines Ärzteverlags Neuland betreten. Wie sah das Verlagskonzept bei der Gründung aus? Welche Ziele wollte man erreichen?}

Das Verlagskonzept ist seit der Gründung unverändert geblieben. Die FMH als Mehrheitsaktionärin von EMH ist ein Akteur im Bereich der medizinischen Publizistik, an dem man nicht vorbeikommt. Das Wesentliche ist dabei, dass es der FMH nicht um einseitige Beeinflussung oder Indoktrination geht. Natürlich nutzt der Zentralvorstand der FMH die Ärztezeitung für Verlautbarungen seiner Politik, die auch als solche deklariert sind. Viel wichtiger ist aber, dass EMH in allen ihren Medien eine absolute redaktionelle Freiheit garantiert und damit einer einseitigen Beeinflussung durch unterschwellige ökonomische oder parteipolitische Interessen der Riegel geschoben wird. Dank seiner Grösse kann sich der Verlag diese redaktionelle Freiheit leisten; die redaktionelle Freiheit garantiert Vielseitigkeit und Qualität. Das ist der eigentliche Kern des verlegerischen Konzepts.

\section{Wie hat sich der junge Verlag in den ersten Jahren nach der Gründung entwickelt?}

Wir begannen ja erst einmal mit der Ärztezeitung und der Schweizerischen Medizinischen Wochenschrift. Die Strategie für die Letztere wurde rasch klar: Aus der alten SchwiWo musste ein rein englischsprachiges Swiss Medical Weekly werden. Als Sprache für wissenschaftliche Publikationen hatte das Deutsche ausgedient. Die SMW wurde von ihren Chefredaktoren Werner Straub und André Perruchoud erfolgreich neu positioniert. 


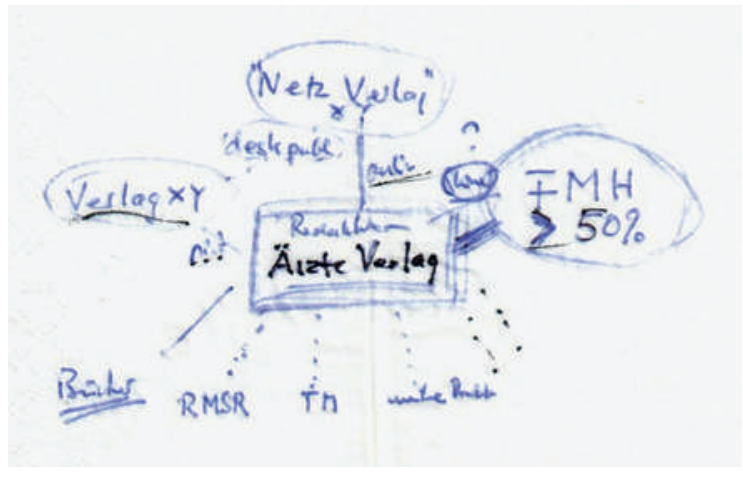

Abbildung 2: Die "Idee EMH» wurde erstmals an einer Tagung am Vierwaldstättersee auf einer Papierserviette visualisiert.

Heute ist sie unter der Leitung von Adriano Aguzzi eine Open-access-Online-Zeitschrift mit internationalem Renommee und erfreut sich monatlich tausender von Zugriffen aus der ganzen Welt. Sie ist heute die einzige schweizerische Zeitschrift, die international rezipiert wird. Die letzte Erfolgsmeldung, der Anstieg des Impact factor auf über 2, bestätigt die hervorragende Arbeit der Redaktion in den letzten Jahren und die Richtigkeit der Strategie.

\section{Hat das von Anfang an funktioniert?}

$\mathrm{Zu}$ Beginn wurde diese Profilierung tatsächlich schlecht aufgenommen. Niedergelassene Ärzte kündigten ihre Abonnements, weil sie nicht englisch lesen wollten, und die Industrie schaltete keine Anzeigen mehr. Das war ein herausfordernder Einstieg.

\section{In welche Richtung wurde die SÄZ entwickelt?}

Die Schweizerische Ärztezeitung sollte aus einer Bleiwüste zum Flaggschiff der gesundheitspolitischen Diskussion werden. Die traditionelle gelbe Farbeder «Brand» vom gelben Heft hatte sich ja in den Köpfen festgesetzt - wollten wir unbedingt behalten, aber den Inhalt durch Fortbildungsartikel und kulturellphilosophische Bereiche des gehobenen «Infotainments» ergänzen (Abb. 3).

\section{Medizinische Fortbildung findet allerdings schon länger nicht mehr in der SÄZ statt ...}

Ja, wir stiessen in der Tat auf ein unvorhergesehenes Hindernis: Was in anderen Ländern üblich ist, nämlich Gesundheitspolitik und seriöse Fortbildung im gleichen Heft zu vereinen, funktionierte bei uns nicht. Es war ernüchternd: Trotz hoher Qualität und Praxisrelevanz wollte niemand Fortbildungsartikel in der SÄZ lesen, mochten sie auch noch so gut sein. Das führte dann dazu, dass wir im Jahr 2001 das Swiss Medical Forum als reine Fortbildungszeitschrift auf den Markt brachten.

\section{Auch mit dieser Entscheidung lagen die Verant- wortlichen aus heutiger Sicht nicht falsch.}

Unter der langjährigen Führung von Reto Krapf hat sich diese Zeitschrift in der Tat zu der anerkannten Fortbildungszeitschrift entwickelt, die die gängige medizinische Praxis unseres Landes widerspiegelt und auch häufig zitiert wird. Aus den drei Zeitschriften SÄZ, SMW und SMF hat sich das Konzept der Kernprodukte entwickelt, die Gesundheitspolitik, Wissenschaft und Fortbildung als zentrale Elemente der Ärzteschaft abdecken.

\section{Gibt es weitere publizistische Meilensteine, die Sie hervorheben würden?}

$\mathrm{Zu}$ den Höhepunkten unter den Produkten, die wir als «Portfolio» bezeichnen, gehört gewiss die Lancierung von PrimaryCare und die hervorragende Kooperation mit den Institutionen der Hausarztmedizin. Gleiches gilt für die Lancierung und Entwicklung von Cardiovascular Medicine.

\section{Wie hat sich das Verhältnis von EMH zur FMH entwickelt?}

Wir waren immer und sind der Verlag der Ärzteschaft, der mehrheitlich der FMH gehört. Allerdings haben auch wir die unterschiedlichen Charaktere der FMH-Präsidenten gespürt. Das heisst, manchmal waren wir näher dran und manchmal weiter weg vom politischen Geschehen.

"Aus den drei Zeitschriften SÄZ, SMW und SMF hat sich das Konzept der Kernprodukte entwickelt, die Gesundheitspolitik, Wissenschaft und Fortbildung als zentrale Elemente der Ärzteschaft abdecken.»

\section{Sie haben als Verwaltungsratspräsident mit drei} FMH-Präsidenten zusammengearbeitet, die gleichzeitig starke Führungspersönlichkeiten sind: Hans Heinrich Brunner, Jacques de Haller und Jürg Schlup. Lassen sich deren Verhältnis zu EMH beziehungsweise die Art der Zusammenarbeit in wenigen Worten auf den Punkt bringen?

Hans Heinrich hatte grossartige strategische, ja visionäre Züge. Er hat EMH ermöglicht und auf den Weg gebracht. Aber wer ihn kannte, weiss, dass er auch manipulativ sein konnte. So versuchte er auch EMH in einzelnen Bereichen zu instrumentalisieren, und das hat im Verwaltungsrat zu heftigsten Kontroversen und schweren Konflikten geführt. Jacques wollte sich vor allem stilistisch von seinem Vorgänger abgrenzen. Er hatte EMH viel Sympathie entgegengebracht und Unterstützung gegeben, engagierte sich intensiv in der Redaktion der SÄZ, aber er hat das Unternehmen gleichzeitig auch be- 
wusst von den politischen Zentren ferngehalten. Diese Distanz zwischen Generalsekretariat und Verlag musste erst wieder überwunden werden durch die aktivere strategische Führung von Jürg Schlup, und mit der Wahl von Urs Stoffel ist nun endlich auch wieder ein Mitglied des ZV im Verwaltungsrat.

\section{Wo steht der Verlag innerhalb der medizinischen Publizistik in der Schweiz mit seinem Zeitschriften- Portfolio und seinem Buchprogramm heute?}

Es klingt fast etwas überheblich, aber wir haben das erreicht, was wir wollten. Im Segment der medizinischen Fachzeitschriften sind wir in der Schweiz Marktführer. Das war vor zwanzig Jahren noch unvorstellbar. Das Buchprogramm könnte selbstverständlich erweitert werden, aber da gilt es auch abzuwägen, was im jeweiligen Sprachraum sinnvollerweise in Buchform publiziert werden muss. Unser strategisches Ziel, ein aktiver Partner in der medizinischen Publizistik zu werden, haben wir aber erreicht!

In welchen Bereichen liegen die grössten Herausforderungen, Gefahren oder Bedrohungen für EMH?

Die grösste Herausforderung liegt nach wie vor in der Sicherstellung der Finanzierung. Die klassischen Geschäftsmodelle der Zeitungen und Zeitschriften stehen überall unter Druck. Eine Substitution durch rein digitale Kommunikationsorgane ist aus vielerlei Gründen kein gangbarer Weg. Es werden sich hier neue Kooperationswege finden, die Synergien schaffen. Ein Beispiel ist etwa die Publikation der extended Abstracts des

«Es klingt fast etwas überheblich, aber wir haben das erreicht, was wir wollten. Im Segment der medizinischen Fachzeitschriften sind wir in der Schweiz Marktführer.»

Weekly im Swiss Medical Forum. Diese Zusammenfassungen ermöglichen einen Hinweis auf die vollständigen Artikel in der Online-Ausgabe, ohne dass ein langes Manuskript in Print publiziert werden müsste.

Wie sehen Sie den Stellenwert des Sockelabonnements für die Kernprodukte, das die Ärztekammer vor einigen Jahren beschlossen hat?

Trotz aller Effizienzanstrengungen geht es nicht ohne einen Abonnementsbeitrag. Die Ärztekammer hat diese Notwendigkeit verstanden. Persönlich bin ich eigentlich der Auffassung, dass dieses bescheidene Sockelabonnement von 50 Franken Teil des regulären Mitgliederbeitrags sein muss und alle Mitgliedergruppen, die die EMH-Produkte beziehen, umfassen sollte.

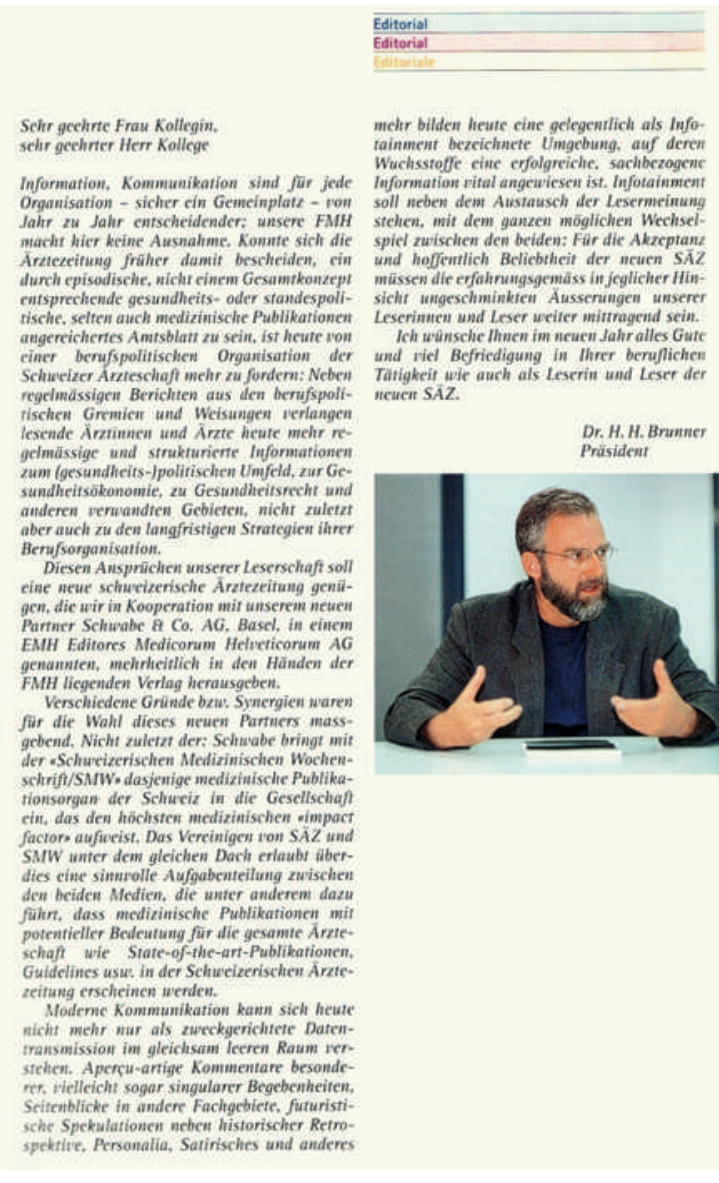

Abbildung 3: In seinem ersten Editorial erklärte Hans Heinrich Brunner das Konzept der «neuen SÄZ».

vergleicht, die wöchentlich dafür geboten werden, der wird leicht verstehen, dass die Finanzierung der EMHProdukte immer noch stark von Fremdfinanzierung abhängig ist. Das ist nicht ohne Risiken.

\section{Wo sehen Sie Entwicklungspotential?}

In diesem Frühjahr haben wir zum ersten Mal eine gemeinsame Nummer von Swiss Medical Forum und Revue Médicale Suisse herausgegeben. Wenn ich mir etwas wünschen würde, dann wäre es das, diese Kooperation weiter zu vertiefen und gemeinsame Synergien zu nutzen. Die Revue Médicale, bei der ja die SMSR massgeblich engagiert ist, ist uns in Geist und Qualität eng verwandt.

\section{Als Chefarzt und Leiter einer grossen medizinischen Klinik brauchen Sie sich auch keine Sorgen über Unterbeschäftigung zu machen. Bleiben Sie der Publizistik dennoch weiterhin verbunden?}

Nun ja, das wird sich zeigen. Auf alle Fälle werde ich EMH aus Distanz auch weiterhin verbunden bleiben und hoffen, dass die «Idee EMH» sich weiterentwickelt und festigt. Ich wünsche dem grossartigen Team jedenfalls allen Erfolg. 